\title{
Impactos da Calibração de Modelo de Velocidade para Conversão Tempo- Profundidade: Um Estudo de Caso
}

\author{
Frank C. Bulhões (Petrobras), Gleidson D. Ferreira (Petrobras), José Fernando Caparica Jr. (Schlumberger)
}

Copyright 2018, SBGf - Sociedade Brasileira de Geofísica

Este texto foi preparado para a apresentação no VIII Simpósio Brasileiro de Geofísica, Salinópolis, 18 a 20 de setembro de 2018 . Seu conteúdo foi revisado pelo Comitê Técnico do VIII SimBGf, mas não necessariamente representa a opinião da SBGf ou Técnico do VIII SimBGf, mas não necessariamente representa a opinião da SBGf ou
de seus associados. É proibida a reprodução total ou parcial deste material para propósitos comerciais sem prévia autorização da SBGf.

\section{Resumo}

On this work we present how the seismic interpretation uncertainties can impact on the construction of the velocity model and consequently on the time-depth conversion. The case study presented is located in the Campos Basin, Brazil. The main objective of this work is to show how the input data and the parameters affect substantially the velocity modeling. The methodology uses different modes and calibration parameters of the velocity model from seismic data interpreted from wells. We present two main approache: one using time-depth tables and another using horizons and geological markers. The data converted to depth are compared to the time data and the geological markers.

\section{Introdução}

As maiores reservas brasileiras de petróleo localizam-se nas bacias de Campos, Santos e Espírito Santo. Essas bacias apresentam marcante tectônica salina e grande potencial exploratório no pré-sal. Com o passar dos anos, novas técnicas de aquisição e processamento foram desenvolvidas de modo a mitigar as incertezas na interpretação sísmica.

A construção do modelo de velocidade para conversão tempo-profundidade é uma das principais etapas na construção do modelo geológico. Dessa forma, o geofísico deve analisar primeiro os pontos importantes tais como: a ordem de grandeza da área, a complexidade estrutural dos objetivos em sub-superfície, o tipo de dado, sua qualidade (e.g. conteúdo de frequências), a variação lateral de velocidades, a disponibilidade de poços para calibração e o ajuste do modelo. O fluxo básico para modelagem de velocidade tem como insumos: velocidade de processamento (tempo ou profundidade), horizontes e dados de poços (tabelas tempo-profundidade, perfis e marcadores geológicos para os respectivos horizontes).

A geometria de aquisição sísmica e 0 tipo de processamento (PSTM, PSDM, migração Kirchhoff, RTM, etc.) apresentam diferentes qualidades de imageamento sísmico e posicionamento dos eventos, impactando diretamente na interpretação sísmica e na amarração de poços, gerando resultados mais ou menos consistentes para o modelo de velocidade (Bulhões et al, 2014).

O processamento de dados sísmicos evoluiu da preparação de dados no domínio do tempo e migração de tempo para imagens de profundidade. Atualmente, a parte de processamento no domínio do tempo consiste principalmente em condicionamento de dados, ou seja, ganho e filtragem, enquanto que o processamento em profundidade consiste em muito mais do que a migração em profundidade (Kessler et al, 2017).

Atualmente, o imageamento sísmico em profundidade desempenha papel central na integração desses processos de processamento de dados sísmicos e interpretação geológica. Após o pré-processamento no domínio do tempo, a construção do modelo geológico em profundidade e o trabalho de interpretação são realizados simultaneamente (Kessler et al, 2017). O fluxo de trabalho do modelo de construção precisa ser ajustado à geologia da área investigada e, portanto, a orientação geológica é a chave para uma construção bem-sucedida do modelo de velocidade.

O modelo final de velocidade tem como objetivos produzir a melhor imagem sísmica (processamento) e converter com precisão os dados sísmicos de tempo para profundidade. Além de obter a imagem em subsuperfície, o modelo de velocidade são entradas para inversão para estimativa de atributos de propriedades físicas das rochas.

A modelagem de velocidade possibilita a utilização de informações de velocidade oriundas da sísmica e do poço, provendo um conjunto de dados mais robusto para uma análise crítica e controle de qualidade. Os critérios para um modelo de velocidade ser robusto, de acordo com Etris et al (2001), são:

1) Modelo deve ser geologicamente consistente;

2) Deve utilizar velocidade apropriadamente detalhada;

3) Deve incorporar todas as informações de velocidade e interpretação disponíveis, ponderando os diferentes tipos de dados apropriadamente (e.g. sísmica vs poços).

Um modelo de velocidade é dito geologicamente consistente, segundo Crabtree et al (2000), quando as velocidades são consistentes com 0 horizonte; a consistência lateral nas velocidades geológicas garante que os efeitos "bulls eye" na calibração com dados de poços sejam evitados; e as amarrações com os poços e em cada linha de interseção sejam consistentes.

A construção de um modelo de velocidade a partir de diversos tipos de dados que apresentam diversos graus de incerteza e resolução, particularmente dados de poço comparados ao dado sísmico (Figura 1). As informações de velocidade na posição do poço podem ser o Vertical Seismic Profile (VSP), levantamento de check shots e perfis sônicos. VSP ou check shots podem ser utilizados diretamente, mas o perfil sônico requer correções de drift ao comparar com o VSP e o check shot do mesmo poço (Reilly, 1993). De uma forma geral, a melhor prática é 
integrar os diferentes tipos de curvas tempo-profundidade (Etris et al, 2001).

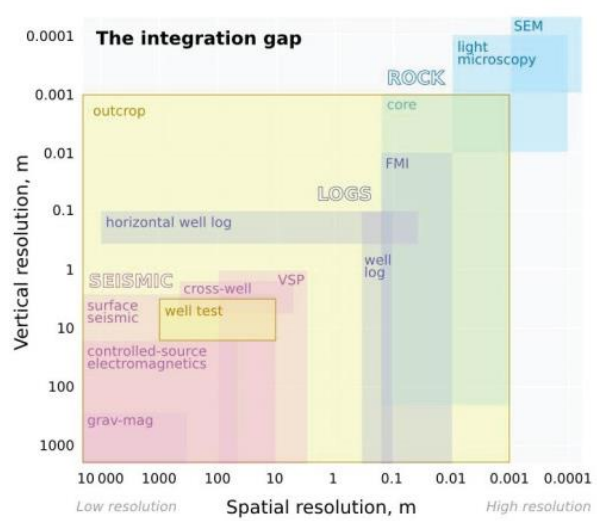

Figura 1: Resolução vertical e lateral para diferentes tipos de métodos e dados geofísicos.

Fonte: Hall (2010)

A tabela tempo-profundidade é utilizada para estimar a profundidade correspondente a cada tempo de reflexão, nas seções de reflexão sísmica. Essa tabela pode ser obtida a partir do perfil sônico do poço ou check shot. A relação entre tempo e profundidade na posição dos poços é obtida associando os eventos obtidos do sismograma sintético do poço com a sísmica em tempo. Pelo modelo convolucional (Rosa, 2010), o sismograma é obtido pela operação matemática da equação 1 .

$$
r(t) * w(t)+n(t)=s(t)
$$

Onde $r(t)$ é a refletividade, $w(t)$ é a wavelet, $n(t) \quad$ o ruído e $s(t)$ o sinal, e o sinal * denota convolução. A refletividade é definida pelos contrastes de impedância (equação 2).

$$
r(t)=\frac{\rho_{i} V_{i}-\rho_{i-1} V_{i-1}}{\rho_{i} V_{i}+\rho_{i-1} V_{i-1}}
$$

Por similaridade, o geocientista correlaciona os eventos em profundidade (poço) com os eventos em tempo (sísmica).

Os dados de poços são medidas "hard" das propriedades físicas em profundidade, e embora não sejam completamente isentos de erros, apresentam menor incerteza no que diz respeito à profundidade, possuindo uma taxa de amostragem da ordem de centímetros. No entanto, dados de poços costumam ser informações geograficamente esparsas, e irregularmente distribuídas, muitas vezes apresentando grande densidade de poços numa pequena região, e pouca ou nenhuma informação em outras regiões. Inclusive o dado de poço apresenta um viés para certas características de propriedade das rochas, pois estão localizadas em altos estruturais e sob condições especiais de acúmulos de hidrocarbonetos ou outros tipos de fluidos. O dado sísmico por outro lado é densamente amostrado e em geral cobre completamente a área de interesse. Ao utilizarmos as informações advindas da sísmica, temos a oportunidade de compensar algumas das limitações de se usar somente os dados de poços. Entretanto, os dados sísmicos são as medidas de tempo numa razão de amostragem maior que a profundidade e a velocidade derivada de poços, e as velocidades de processamento geradas a partir da sísmica são velocidades de imageamento, e não de propagação vertical, como a dos poços. Para combinar o dado "hard" dos poços, com menor incerteza vertical e pouco amostrada espacialmente, com o dado "soft" do dado sísmico, com maior incerteza e densamente amostrado espacialmente, e honrando o dado de maior certeza e confiabilidade do dado de poço, podemos utilizar ferramentas geostatísticas.

A geoestatística leva em consideração a distância entre as observações (poços) que leva em consideração a localização geográfica e a dependência espacial das amostras (Camargo, 1999). As resoluções lateral e vertical variam na escala de ordem de grandeza conforme o objetivo é integrar essas diferentes fontes de dados de modo a obter uma descrição da profundidade em subsuperfície com uma medida de incerteza.

\section{Metodologia/ Problema Investigado}

O trabalho é um estudo de caso localizado na Bacia de Campos, com aproximadamente $700 \mathrm{~km}^{2}$, em um ambiente tectônico com eventos erosivos, onde as incertezas nas interpretações sísmicas impactam na amarração poço-sísmica e, consequentemente, na modelagem de velocidade e conversão para profundidade.

No trabalho para ajuste do modelo de velocidade para conversão tempo-profundidade utilizaram-se sete horizontes, sísmica PSDM e modelo de velocidade, advindos do processamento, 17 poços com tabelas tempo-profundidade e sete marcadores geológicos referentes aos respectivos horizontes interpretados, volumes de pseudo-impedância-P e -S.

Entre os desafios desse trabalho destacam-se incertezas de ordem geológica e dos dados geofísicos. Entre os dados geofísicos está a ausência de perfilagem na seção rasa, impossibilitando uma correlação tempoprofundidade e tendo como únicas informações nessa parte a velocidade sísmica de migração. Associados a isso, os horizontes referentes aos eventos mais rasos apresentam maior incerteza de mapeamento devido à ausência de seção e a sobreposição de discordâncias e eventos erodidos.

Diante das incertezas supracitadas, trabalhou-se com três cenários de ajuste e calibração do modelo de velocidade, variando-se o tipo de ajuste marcadoreshorizontes, calibração tabelas tempo-profundidade e diferentes raios de influência, conforme Figura 2.

\begin{tabular}{|l|c|c|c|}
\hline \multicolumn{1}{|c|}{ Parâmetros } & Cenário 1 & Cenário 2 & Cenário 3 \\
\hline $\begin{array}{l}\text { Ajuste } \\
\text { (Tabelas/Marcadores) }\end{array}$ & $\begin{array}{c}\text { Marcadores } \\
\text { Horizontes }\end{array}$ & $\begin{array}{c}\text { Tabelas TP } \\
\text { Kriging com } \\
\text { deriva externa }\end{array}$ & $\begin{array}{c}\text { Tabelas TP } \\
\text { Kriging com } \\
\text { deriva externa }\end{array}$ \\
\hline Elipse de Anisotropia & $5 \mathrm{~km} \times 5 \mathrm{~km}$ & $5 \mathrm{~km} \times 5 \mathrm{~km}$ & $2 \mathrm{~km} \times 2 \mathrm{~km}$ \\
\hline
\end{tabular}

Figura 2: Cenários de calibração do modelo de velocidade. 
Os critérios para controle de qualidade e validação do modelo são:

1) Análise da conversão dos volumes sísmicos e de pseudo-impedância-P e -S advindas da inversão, comparando-se as correlações obtidas na conversão para profundidade em cada um dos três cenários; e

2) Conversão dos mapas de tempo para profundidade e calculando os resíduos em relação aos respectivos marcadores $(\delta z)$, adotando o critério de Widess como parâmetro (Maul et al, 2013).

A resolução sísmica foi calculada a partir do volume de frequência dominante $\left(\mathfrak{f}_{\mathrm{dom}}\right)$ extraído do dado sísmico e do modelo de velocidade calibrado. Pelo critério de Widess (1973), a resolução vertical sísmica é dada por:

$$
\frac{\lambda}{4}=\frac{V}{4 f_{\text {dom }}}
$$

onde valores de $\delta z \leq N 4$ implicam em erros abaixo da resolução sísmica, indicando assim um resultado aceitável de acordo com a limitação do método sísmico.

\section{Resultados}

A comparação entre os três cenários apresentou que no quesito de menor resíduo da profundidade do horizonte comparada ao marcador na posição do poço, o cenário 1 apresentou a menor diferença, como observa-se na secção sísmica da figura 3. Por outro lado, quando analisam-se a conversão dos volumes de pseudoimpedância-p advinda da inversão verifica-se uma forte diminuição da correlação (Figura 4).
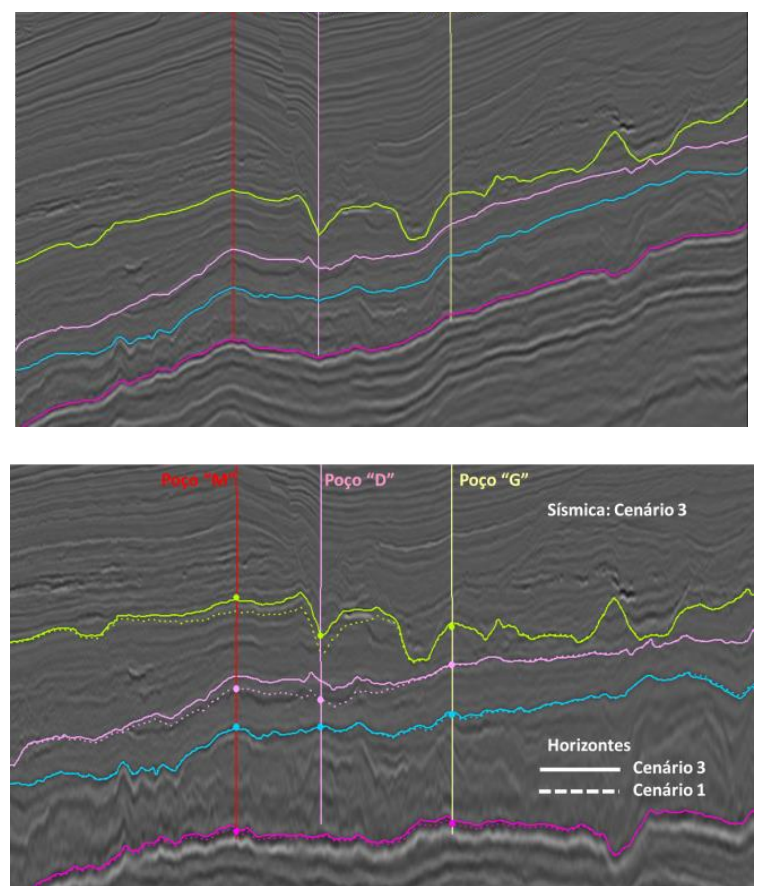

Figura 3: Na figura superior, seção arbitrária em tempo e na figura inferior a respectiva seção com os horizontes convertidos para profundidade pelos cenários 1 e 3 . Sísmica convertida pelo cenário 3.

\begin{tabular}{|c|c|c|c|c|c|c|}
\hline \multicolumn{2}{|c|}{ Horizonte 5 } & \multicolumn{2}{c|}{ Cenário 1 } & \multicolumn{2}{c|}{ Cenário 3 } & \\
\hline Poço & $\begin{array}{c}\text { Correlação Ip - } \\
\text { Tempo }\end{array}$ & $\begin{array}{c}\text { Correlação } \\
\text { IP }\end{array}$ & $\begin{array}{c}\text { Dif Hor- } \\
\text { Marker (m) }\end{array}$ & $\begin{array}{c}\text { Correlação } \\
\text { IP }\end{array}$ & $\begin{array}{c}\text { Dif Hor- } \\
\text { Marker (m) }\end{array}$ & 1/4 (m) \\
\hline B & 0,57 & 0,0 & 3,67 & 0,56 & 56,17 & 39,08 \\
\hline C & 0,83 & $-0,23$ & 0,48 & 0,79 & 21,30 & 38,00 \\
\hline D & 0,79 & 0,79 & 0,53 & 0,77 & 0,47 & 38,63 \\
\hline E & 0,82 & $-0,03$ & 5,04 & 0,70 & 5,04 & 25,75 \\
\hline F & 0,77 & 0,69 & 0,04 & 0,76 & 4,83 & 28,04 \\
\hline G & 0,90 & 0,65 & 0,03 & 0,90 & 11,71 & 28,24 \\
\hline H & 0,84 & 0,09 & 1,38 & 0,71 & 8,18 & 25,31 \\
\hline I & 0,91 & $-0,12$ & 0,56 & 0,91 & 3,58 & 31,52 \\
\hline J & 0,81 & 0,59 & 0,08 & 0,80 & 6,31 & 37,11 \\
\hline K & 0,76 & $-0,28$ & 8,83 & 0,79 & 14,93 & 46,74 \\
\hline L & 0,85 & 0,18 & 0,10 & 0,80 & 10,13 & 36,81 \\
\hline M & 0,66 & 0,40 & 0,68 & 0,47 & 18,99 & 30,97 \\
\hline
\end{tabular}

Figura 4: Tabela com as correlações do volume de Impedância-P com a Pseudo-Impedância obtida da inversão, convertidos para profundidade pelos modelos calibrados com marcadores e pelas tabelas (Coluna "Correlação Ip"). Também constam as diferenças do horizonte "H5" (convertido para profundidade pelos dois modelos) comparados aos respectivos marcadores nos poços.

A correlação entre os perfis de pseudo-impedância e as respectivas impedâncias convertidas em profundidade não é preservada na maioria dos poços.

Quatro poços destacam-se em termos de comportamento diante dos três cenários expostos (Figura 5):

1) Poço "D" que não há alterações significativas: Os marcadores interpretados neste poço e os perfis sônico e Rhob foram utilizados no processamento da sísmica PSDM.

(2) Poços "G" e "I": Nota-se neste poço que o ajuste dos horizontes aos marcadores (Cenário 1) destrói a relação tempo-profundidade. O raio de influência não interferiu na calibração por serem poços afastados em relação aos demais.

(3) Poço "K", no qual é observado o quão substancialmente interfere na conversão a utilização dos horizontes e marcadores na calibração. O raio de influência também interfere fortemente devido a poços a distâncias menores a esses raios.

Pelos cenários apresentados e discorridos, o cenário 3 apresentou-se o mais adequado para conversão tempoprofundidade das sísmicas e horizontes por:

i) Apresentar uma diferença entre os mapas convertidos e marcadores (na faixa do limite da resolução sísmica);

ii) Melhor preserva a correlação dos volumes de pseudoimpedância ( $\mathrm{P}$ e S) obtidos na inversão. 


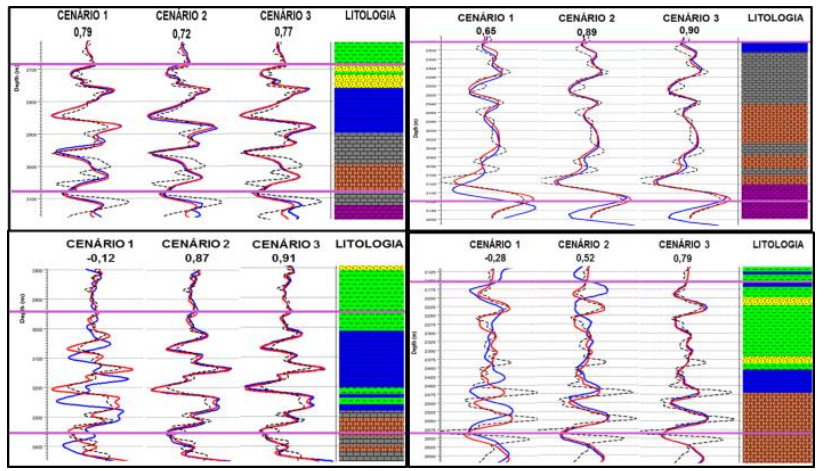

Figura 5: Perfis de impedância-p banda passante, pseudo-impedância em tempo (tabela TP) e convertido para profundido pelos três cenários. (a) Poço "D". (b) Poço "G". (c) Poço "I": (d) Poço "K". Cortesia do geofísico Erick Talarico.

No cenário 1 , construiu-se modelo com calibração às tabelas TP e marcadores, que produziram mapas em profundidade que honram os marcadores, mas implicaram em um modelo de velocidade com valores anômalos e distorções na sísmica convertida para profundidade. No controle de qualidade nas seções sísmicas próximas aos poços observaram-se interpretações dúbias que permitiam interpretação em diversos cenários.

Restringindo a calibração com os marcadores aos poços em que a interpretação não apresenta dubiedade, os efeitos anômalos na velocidade e as distorções na sísmica convertida para profundidade não ocorrem. Um aspecto importante a ser considerado é a perda da amarração tempo-profundidade a partir do sismograma sintético. Em termos qualitativos o ajuste em profundidade dos horizontes aos marcadores apresentam resultados satisfatórios comparados à resolução sísmica, considerando-se a escala de resolução sísmica pelo critério de Widess (N/4). Por outro lado quando se analisa a conversão dos volumes de pseudo-Impedância- $P$ advinda da inversão verifica-se uma forte diminuição na correlação com a Impedância-P do sintético dos poços.

\section{Discussão e Conclusões}

Durante a execução da conversão tempo-profundidade, foi notável a disparidade nas seções e horizontes resultantes, dada as variações em velocidade e nas interpretações iniciais de horizontes em tempo. A presença de incertezas e o estudo de sensibilidade são, por vezes, ignorados no contexto da exploração, e nestes trabalhos foi possível constatar que eles são necessários para identificar a causa de eventuais distorções.

O caso em estudo mostrou que, ao se confiar plenamente na amarração de marcadores, podem-se criar artefatos e distorções nos resultados convertidos, ainda que aparentemente os resultados estejam satisfatórios. Neste caso, a disparidade se mostrou evidente quando comparados os volumes de impedância, e nos levou a questionar: estariam estes marcadores corretamente amarrados ou os horizontes nas regiões dos poços apresentam uma margem considerável de incerteza. As interpretações estão em revisão para essa questão ser respondida.

Como produto deste projeto, podem ser acrescidos critérios os itens necessários para efetuar o controle de qualidade de um modelo de velocidade: velocidades são geologicamente coerentes; ausência de marcas de calibração no modelo; ausência de deformações que são geologicamente incoerentes no dado sísmico após conversão para profundidade; ausência de inversões de estruturas em mapas convertidos para profundidade; e comparação entre horizontes convertidos para profundidade e respectivos marcadores.

Conclui-se que diante de cenários destoantes, a interpretação sísmica influencia de forma substancial os resultados da modelagem final. Pequenas variações nos dados de entrada podem levar a geração de estruturas inexistentes e velocidades anômalas sem significado geológico. Além disto, observa-se que em alguns casos é necessário se abster de alguns poços e seus marcadores geológicos em áreas complexamente estruturadas e com fortes variações laterais de velocidades, para que se consiga um melhor ajuste do modelo na conversão de horizontes em profundidade.

\section{Agradecimentos}

Agradecemos à Petrobras por permitir a publicação deste trabalho. Agradecemos ao nosso gerente Francisco Aquino pelo apoio na publicação do trabalho.

\section{Referências}

Al-Chalabi, 1994. M. Seismic velocity - A critique. First Break, 12, p. 589-596.

Bulhões, F. C. et al., 2014. Fluxo para construção do Modelo de Velocidade Regional da Bacia de Campos. VI Simpósio Brasileiro de Geofísica da SBGF. Porto Alegre (RS), Brasil.

Bulhões, F. C. et al., 2017. Case study: On the impact of interpretation uncertainty over velocity modeling for timedepth conversion. 15th International Congress of Brazilian Geophysical Society \& EXPOGEF, Rio de Janeiro, Brazil.

Camargo, E., Monteiro, A.M.V., Felgueiras, C.A., Fuks, S.D., 1999. Integração de Geoestatística e Sistemas de Informação Geográfica: Uma Necessidade. In GIS Brasil 99.

Crabtree, N. J., Etris, E. L., Eng, J., Brewer, G., Dewar, J., 2000. Geologically consistent seismic processing velocities improve time to depth conversion. Geological Association of Canada.

Duarte, O. O., 2010. Dicionário Enciclopédico InglêsPortuguês de Geofísica e Geologia, Sociedade Brasileira de Geofísica.

Etris, E. L., Crabtree, N. J., Dewar, J., 2001. True Depth Conversion: More than a pretty picture. CSEG Recorder.

Fetter, M., Daltro, D., Lafferrieire, F. \& Souza JR., O. G., 2004. Filtragem de Velocidades Intervalares Através de Krigagem Fatorial. SIMGEF (Simpósio de Geofísica da Petrobras). Aracaju, Brasil. 
Kessler, D., Kosloff, D., Codd J., e Bartana, A., 2017. Depth Imaging - More than PSDM. 15th International Congress of the Brazilian Geophysical Society \& EXPOGEF, Rio de Janeiro, Brazil.

Jones, I. F., 2008. SPG Hyderabad Key-Note Address: Non-uniqueness and ambiguity in velocity model building. 7th International Conference \& Exposition on Petroleum Geophysics. Hyderabad.

Liner, C., 2016. Elements of 3D seismology. Society of Exploration Geophysicists.

Luton, L. C. e Prieto, C., 2000. Accurate Depth Conversions Reduce Risk. Gulf Coast Association of Geological Societies Transactions, Volume L.

Maul, A. R. et al., 2013. Modelagem Geológica de Velocidades Sísmicas Utilizando o Gocad - Material interno Petrobras.

Nery, G. G., 2013. Perfilagem Geofísica em Poço Aberto - Fundamentos Básicos com ênfase em petróleo. Sociedade Brasileira de Geofísica. Rio de Janeiro.

Reilly, M., 1993. Integration of well and seismic data for $3 \mathrm{D}$ velocity model building, First Break, v. 11, n. 6, p. 247260.

Rosa, A. L. R., 2010. Análise do Sinal Sísmico - 1aㅗ edição. Rio de Janeiro - Sociedade Brasileira de Geofísica (SBGF).

Ross, W. S., 1994. The velocity-depth ambiguity in seismic traveltime data. Geophysics, v. 50 , n. 5 , p. 830843.

Shelandera, D., Smithb, V., Leetaruc, H., Willd, r. \& Couëslane, M., 2014. The Evolution and Effect of the Velocity Model on Various Aspects of Surface Seismic Data Analysis. Energy Procedia n. 63, p. 4374 - 4384.

Sheriff, R. E., Geldard, L. P., 1995. Exploration Seismology. Second Edition. Cambridge University Press.

Tieman, H.J., 1994. Investigating the velocity-depth ambiguity of reflection traveltimes. Geophysics, v. 59, n. 11 , p. 1763-1773.

Widess, M. B., 1973. How thin is a thin bed. Geophysics, v. 38, p. $1176-1254$.

Yilmaz, O., 2008. Seismic Data Analysis. Processing, Inversion, and Interpretation of Seismic Data. Society of Exploration Geophysicists. Eletronic Edition. 